\title{
基于电子舌技术的氨基酸与密码子关系的初步研究
}

\author{
赵钊 $a$ 程时文 $b$ 毛岳忠 $b$ 应见喜 $c$ 田师一 $*, b$ 赵玉芬*, $a, c$ \\ ( ${ }^{a}$ 厦门大学 化学化工学院 福建省化学生物学重点实验室 厦门 361005) \\ ( $b$ 浙江工商大学 食品与生物工程学院 杭州 310018) \\ ( 宁波大学 新药技术研究院 宁波 315211)
}

\begin{abstract}
摘要 自从首个遗传密码子被破译以来, 密码子与所表达的氨基酸之间的关系一直吸引了许多关注. 本研究从氨基 酸、核苷和三偏磷酸钠的反应体系出发，用电子舌检测反应前后体系变化，考察核苷对氨基酸成肽反应的影响，试图找 到氨基酸与密码子的联系. 结果表明，大部分氨基酸的密码子或反密码子中间位核苷对反应的促进作用最大，这种结 果可能是因为核苷的碱基与氨基酸的侧链基团之间的某种弱相互作用导致的. 相应地，亮氨酸和异亮氨酸之间的结果 差异更加佐证了上述假设. 这种弱相互作用有望成为研究密码子识别氨基酸的关键突破, 为生命起源的研究提供新方 法.
\end{abstract}

关键词 密码子; 氨基酸; 电子舌; 核苷; 生命起源

\section{Primary Research of the Relationship between Genetic Codons and Amino Acids Based on the Technology of Electronic Tongue}

\author{
Zhao Zhao $^{a} \quad$ Shiwen Cheng ${ }^{b} \quad$ Yuezhong Mao $^{b}$ Jianxi Ying $^{c} \quad$ Shiyi Tian $^{*, b} \quad$ Yufen Zhao*,a,c $^{*}$ \\ ( ${ }^{a}$ College of Chemistry and Chemical Engineering, and the Key Laboratory for Chemical Biology of Fujian Province, \\ Xiamen University, Xiamen 361005, China) \\ ( ${ }^{b}$ School of Food Science and Biotechnology, Zhejiang Gongshang University, Hangzhou 310018, China) \\ ( ${ }^{c}$ Institute of Drug Discovery Technology, Ningbo University, Ningbo 315211, China)
}

\begin{abstract}
The relationship between genetic codons and amino acids has attracted much attentions since the discovery of first genetic codon. Although we have known that relationship of codons and amino acids clearly, even some rare amino acids also have their codons in certain creatures, we do not know the real principle that how a codon detertmine an amino acid. In fact, the codon is a little far from amino acid in a tRNA. With many efforts, we have not detect the secret of codon. Before we used nuclear magnetic resonance (NMR) and high resolution mass spectrum (HRMS) to detect the product of reaction of amino acids, nucleosides and sodium trimetaphosphate to find out the relationship between amino acid and nucleoside. But the productivity was too low to determine. Electronic tongue is a machine used to determine the whole variant of a solution, especially in food industry, it could determine very subtle variant. So we use it to determine the whole variant of our system. In this work, we reported the reactions between amino acids, nucleosides and sodium trimetaphosphate. Each ingredient was $0.0008 \mathrm{~mol} / \mathrm{L}$ and $\mathrm{pH}$ was adjusted to $11 \mathrm{by} \mathrm{NaOH}$, then reacted at $50{ }^{\circ} \mathrm{C}$ for $48 \mathrm{~h}$. The reacted mixture was cooled down to room temperature and detected by electronic tongue. Also, the samples that were freshly prepared were detected by electronic tongue to be original data. The overall changes of the reaction mixtures were characterized by the average of Euclidean Distance. The results show that the nucleosides in middle of codon or anticodon displayed the greatest effect on the reactions. The results also indicate that there are significant interrelations between amino acids and nucleosides, which was also proved by the difference between the results of leucine and isoleucine. In summary, the results may open a new avenue to the study of the relationship between genetic codens and amino acids and origin of life.

Keywords codon; amino acid; electronic tongue; nucleoside; origin of life
\end{abstract}

\section{1 引言}

自从 20 世纪 60 年代 Matthaei 和 Nirenberg ${ }^{[1]}$ 破译了 首个遗传密码子以来, 人们一直对遗传密码子与氨基酸 的关系保持着浓厚的研究兴趣. 关于这一关系的理论研 究, 目前主要有 $\mathrm{Crick}^{[2]}$ 提出的偶然冻结理论(Frozen accident), 认为密码子与氨基酸的对应关系只是一种偶 然事件导致的，并在长期的进化过程中被固定了下来; Woese 等 ${ }^{[3]}$ 提出的立体化学相互作用理论(Steric interaction), 认为遗传密码子与相对应的氨基酸之间有着立体 化学的作用. 其他影响较大的还有 Wong ${ }^{[4]}$ 提出、Di Giulio $^{[5]}$ 发展的氨基酸-密码子共进化论(Co-evolution);

\footnotetext{
*E-mail: tianshiyi@zjsu.edu.cn; yfzhao@xmu.edu.cn

Received July 16, 2021; published September 15, 2021.

Project supported by Technology and Engineering Center for Space Utilization, Chinese Academy of Sciences (No. YYWT-0901-EXP-16).

项目受中国科学院空间科学与应用总体部(No. YYWT-0901-EXP-16)资助.
} 
Eigen 等[6]提出的试管选择理论(In vitro selection)等. 这 些理论虽有一定的道理, 但是都未能很好地独立解释密 码子与氨基酸的关系，且没有试验数据可以支撑.

从密码子表中, 研究者们发现, 大部分情况下，同 一个氨基酸对应的几个密码子, 前两位都是一样的, 如 果最后一位改变, 一般也是变成极性类似的氨基酸 $($ 表 1).

\section{表 1 密码子表}

Table 1 Codon table

\begin{tabular}{cccccc}
\hline & U & C & A & G & \\
\hline \multirow{4}{*}{ U } & Phe & Ser & Tyr & Cys & U \\
& Phe & Ser & Tyr & Cys & C \\
& Leu & Ser & STOP & STOP & A \\
& Leu & Ser & STOP & Trp & G \\
\hline \multirow{4}{*}{ C } & Leu & Pro & His & Arg & U \\
& Leu & Pro & His & Arg & C \\
& Leu & Pro & Gln & Arg & A \\
& Leu & Pro & Gln & Arg & G \\
\hline \multirow{6}{*}{ A } & Ile & Thr & Asn & Ser & U \\
& Ile & Thr & Asn & Ser & C \\
& Ile & Thr & Lys & Arg & A \\
& Met & Thr & Lys & Arg & G \\
\hline \multirow{3}{*}{ G } & Val & Ala & Asp & Gly & U \\
& Val & Ala & Asp & Gly & C \\
& Val & Ala & Glu & Gly & A \\
& Val & Ala & Glu & Gly & G \\
\hline
\end{tabular}

Lagerkvist $^{[7]}$ 发现，在试管试验里，对一些对应了 4 个密码子的氨基酸(如丙氨酸 Ala), 只用一种转移 RNA (tRNA) 就可以将所有的密码子读出. 基于此, “ 3 中读 2 ” (three-out-of-two) 的理论被提出, 一般认为密码子前两 位的核苷可以决定氨基酸的种类, 第三位核苷的影响较 小. 同时, 对于有 6 个密码子的氨基酸(亮氨酸 Leu 和精 氨酸 Arg), 它们密码子中间位的核苷是不变的. 因此一 般认为密码子中第二位核苷对氨基酸的种类起着决定 性的作用，第一位次之.

最近, 我们研究发现, 氨基酸在核苷和三偏磷酸钠 $\left(\mathrm{P}_{3} \mathrm{~m}\right)$ 存在下, 可以在弱碱性环境下生成二肽 ${ }^{[8-11]}$. 基于 这个研究的发现以及 “ 3 中读 2 ” 的思想, 我们尝试探索 单个核苷对氨基酸成肽的影响的差别, 以此来找到密码 子与氨基酸之间这种对应关系形成的原因. 但是由于单 个核酸对氨基酸成肽产率影响的差别较小, 采用常规仪 器进行检测受到限制.

电子舌系统 ${ }^{[12-14]}$ (Electronic tongue)是一种以非特异 性、低选择性传感器阵列来模拟生物系统舌头的味蕾细 胞, 对不同 “味道” 的被测溶液进行感应检测, 以信号 激发及采集系统来替代人类神经感觉系统, 将味觉物质 信息转换成不同的电化学信号, 并传递给电脑的模式识 别系统, 而电脑相当于人的大脑, 通过对电信号进行特 征提取从而对不同溶液间的整体差异特征进行判别的 智能仿生设备. 在弱碱性的条件下，当往氨基酸中加入
了核苷和三偏磷酸钠后，氨基酸会与加入的物质发生反 应形成二肽，当电子舌传感器阵列接触到氨基酸反应前 后的溶液时, 由于反应前后氨基酸溶液整体性质的差异 不同, 因此, 电子舌的传感器阵列所产生的电化学指纹 信号图谱也会存在一定的差异, 此时, 再结合多元统计 算法将信号图谱间的差异程度转换为量化的差别度值, 即可用于表征氨基酸溶液反应前后的整体差异程度. 基 于此，电子舌非常适合氨基酸密码子反应体系的检测， 即可以通过比较反应溶液前后的差别度，表征不同反应 体系的进行程度. 差别度越大, 则说明反应进行程度越 大. 本文从氨基酸、核苷和 $\mathrm{P}_{3} \mathrm{~m}$ 的成肽反应出发, 将反 应前后的样品通过电子舌检测，并通过欧氏距离平均 值 ${ }^{[15]}$ 计算出反应前后的差别度, 探索氨基酸与密码子 的关系.

\section{2 结果与讨论}

\section{1 不同核苷对反应促进作用的分析}

本实验设计氨基酸、核苷和三偏磷酸钠的反应体系, 将刚配好的溶液和反应 $48 \mathrm{~h}$ 后的溶液分别采用电子舌 进行检测, 并计算差别度. 同时, 利用欧氏距离平均值 作为样品溶液的差别度的量化表征值. 差别度越大, 代 表体系变化越大，以此可以比较不同核苷对同一氨基酸 的影响. 实验结果如下(表 2).

从表 2 来看, 加入密码子中间位的核苷对反应促进 最大的有 7 个氨基酸, 即为: 谷氨酸(Glu)、组氨酸(His)、 甲硫氨酸(Met)、苯丙氨酸 $(P h e) 、$ 丝氨酸 $(\mathrm{Ser}) 、$ 色氨酸 (Trp)和酪氨酸(Tyr), 本研究将其归类为遵循密码子规 则的氨基酸. 加入反密码子中间位的核苷对反应促进最 大的有 9 个, 即为: 丙氨酸(Ala)、精氨酸(Arg)、天冬氨 酸(Asp)、谷氨酰胺 $(\mathrm{Gln})$ 、异亮氨酸(Ile)、赖氨酸(Lys)、 丝氨酸(Ser)、苏氨酸(Thr)和脯氨酸(Pro), 本研究将其归 类为遵循反密码子规则的氨基酸. 还有 5 个氨基酸反应 进行程度最大的既不是密码子中间位，也不是反密码子 中间位, 即为: 天冬酰胺(Asn)、甘氨酸(Gly)、亮氨酸 (Leu)、缅氨酸(Val)和半胱氨酸(Cys), 但它们若以变化第 二大的核苷来看，都遵循密码子规则. Ser 中间位密码子 有两种，因此结果为两者都算.

氨基酸成肽反应的可能历程如下(图 1). 反应中, 氨 基酸首先与 $\mathrm{P}_{3} \mathrm{~m}$ 反应形成三磷酰化氨基酸 ( $N$-Triphosphoryl Amino Acid), 脱掉一个焦磷酸 (Pyrophosphate, PPi), 且环化形成环酰基氨基磷酸酯-1 (Cyclic Acylphosphoramidate-1, CAPA-1), 而 CAPA 已被 证明可能是氨基酸成肽过程中的中间体; 接着核苷的羟 基进攻 CAPA-1, 开环形成酰胺-核苷酸 (Nucleotide Amidate, AA- $N$-NMP); 随后, AA- $N$-NMP 再次环化形成 环酰基氨基磷酸酯-2 (Cyclic Acylphosphoramidate-2, CAPA-2), 然后它被另一分子的氨基酸的氨基进攻, 开 环生成核苷酸 - 二肽 (Nucleotide-Dipeptide, NMP- 
Dipeptide), NMP-Dipeptide 可以水解得到二肽 (Dipeptide)和核苷酸(Nucleotide, NMP) ${ }^{[9]}$.

表 2 差别度计算结果

Table 2 Calculations of Dis3

\begin{tabular}{|c|c|c|c|}
\hline & 密码子 & 差别度结果 & 计算数值 ${ }^{a}$ \\
\hline \multirow[t]{2}{*}{ Ala } & $\mathrm{GCA} / \mathrm{U} / \mathrm{G} / \mathrm{C}$ & $\mathrm{G}>\mathrm{U}>\mathrm{C}>\mathrm{A}$ & $\mathrm{A}: 24.14(0.78) / \mathrm{C}: 26.12(0.16)$ \\
\hline & & & G:27.04(0.08)/U:26.34 (0.11) \\
\hline \multirow[t]{2}{*}{ Arg } & $\mathrm{CGA} / \mathrm{U} / \mathrm{G} / \mathrm{C}, \mathrm{AGA} / \mathrm{G}$ & $\mathrm{C}>\mathrm{G}>\mathrm{A}>\mathrm{U}$ & $\mathrm{A}: 26.33(0.13) / \mathrm{C}: 27.39(0.07)$ \\
\hline & & & G:26.68(0.14)/U:26.00(0.29) \\
\hline \multirow[t]{2}{*}{ Asn } & $\mathrm{AAU} / \mathrm{C}$ & $\mathrm{C}>\mathrm{A}>\mathrm{U}>\mathrm{G}$ & $\mathrm{A}: 27.53(0.23) / \mathrm{C}: 28.08(0.07)$ \\
\hline & & & G:24.35(0.25)/U:27.02(0.18) \\
\hline \multirow[t]{2}{*}{ Asp } & $\mathrm{GAU} / \mathrm{C}$ & $\mathrm{U}>\mathrm{G}>\mathrm{A}>\mathrm{C}$ & $\mathrm{A}: 27.25(0.44) / \mathrm{C}: 27.00(0.42)$ \\
\hline & & & $\mathrm{G}: 27.53(0.42) / \mathrm{U}: 28.26(0.16)$ \\
\hline \multirow[t]{2}{*}{ Cys } & $\mathrm{UGC} / \mathrm{U}$ & $\mathrm{A}>\mathrm{G}>\mathrm{U}>\mathrm{C}$ & $\mathrm{A}: 26.37(0.16) / \mathrm{C}: 22.76(0.78)$ \\
\hline & & & G:24.81(0.86)/U:23.90(0.31) \\
\hline \multirow[t]{2}{*}{ Gln } & $\mathrm{CAA} / \mathrm{G}$ & $\mathrm{U}>\mathrm{A}>\mathrm{C}>\mathrm{G}$ & $\mathrm{A}: 27.73(0.33) / \mathrm{C}: 27.61(0.12)$ \\
\hline & & & G:27.54(0.26)/U:27.94(0.17) \\
\hline \multirow[t]{2}{*}{ Glu } & GAA/G & $\mathrm{A}>\mathrm{C}>\mathrm{G}>\mathrm{U}$ & $\mathrm{A}: 27.71(0.28) / \mathrm{C}: 27.52(0.12)$ \\
\hline & & & G:27.09(0.23)/U:26.17(0.22) \\
\hline \multirow[t]{2}{*}{ Gly } & $\mathrm{GGA} / \mathrm{U} / \mathrm{G} / \mathrm{C}$ & $\mathrm{A}>\mathrm{G}>\mathrm{C}>\mathrm{U}$ & A:26.73(0.60)/C:25.98(0.34) \\
\hline & & & G:26.07(0.37)/U:25.66(0.10) \\
\hline \multirow[t]{2}{*}{ His } & $\mathrm{CAU} / \mathrm{C}$ & $\mathrm{A}>\mathrm{C}>\mathrm{U}>\mathrm{G}$ & $\mathrm{A}: 27.33(0.48) / \mathrm{C}: 27.24(0.41)$ \\
\hline & & & $\mathrm{G}: 24.19(0.60) / \mathrm{U}: 26.32(0.59)$ \\
\hline \multirow[t]{2}{*}{ Ile } & $\mathrm{AUA} / \mathrm{U} / \mathrm{C}$ & $\mathrm{A}>\mathrm{C}>\mathrm{G}>\mathrm{U}$ & A:28.18(0.07)/C:27.92(0.28) \\
\hline & & & G:27.28(0.30)/U:26.62(0.36) \\
\hline \multirow[t]{2}{*}{ Leu } & $\mathrm{CUA} / \mathrm{U} / \mathrm{G} / \mathrm{C}$ & $\mathrm{G}>\mathrm{U}>\mathrm{A}>\mathrm{C}$ & $\mathrm{A}: 26.67(0.23) / \mathrm{C}: 26.02(0.65)$ \\
\hline & & & $\mathrm{G}: 27.93(0.10) / \mathrm{U}: 26.97(0.37)$ \\
\hline \multirow[t]{2}{*}{ Lys } & $\mathrm{AAA} / \mathrm{G}$ & $\mathrm{U}>\mathrm{A}>\mathrm{C}>\mathrm{G}$ & $\mathrm{A}: 26.63(0.54) / \mathrm{C}: 26.25(0.09)$ \\
\hline & & & G:21.47(0.11)/U:26.97(0.34) \\
\hline \multirow[t]{2}{*}{ Met } & AUG & $\mathrm{U}>\mathrm{C}>\mathrm{A}>\mathrm{G}$ & $\mathrm{A}: 25.19(0.31) / \mathrm{C}: 25.44(0.98)$ \\
\hline & & & $\mathrm{G}: 24.96(0.30) / \mathrm{U}: 26.69(0.27)$ \\
\hline \multirow[t]{2}{*}{ Phe } & $\mathrm{UUA} / \mathrm{U} / \mathrm{G} / \mathrm{C}$ & $\mathrm{U}>\mathrm{G}>\mathrm{C}>\mathrm{A}$ & $\mathrm{A}: 23.58(0.55) / \mathrm{C}: 24.28(0.49)$ \\
\hline & & & $\mathrm{G}: 26.00(0.20) / \mathrm{U}: 26.16(0.79)$ \\
\hline \multirow[t]{2}{*}{ Pro } & $\mathrm{CCA} / \mathrm{U} / \mathrm{G} / \mathrm{C}$ & $\mathrm{G}>\mathrm{A}>\mathrm{C}>\mathrm{U}$ & $\mathrm{A}: 26.85(0.79) / \mathrm{C}: 26.60(0.38)$ \\
\hline & & & G:27.12(0.12)/U:26.53(0.79) \\
\hline \multirow[t]{2}{*}{ Ser } & $\mathrm{UCA} / \mathrm{U} / \mathrm{G} / \mathrm{C}, \mathrm{AGU} / \mathrm{C}$ & $\mathrm{G}>\mathrm{A}>\mathrm{C}>\mathrm{U}$ & A:28.77(0.03)/C:28.66(0.49) \\
\hline & & & G:29.23(0.12)/U:28.59(0.34) \\
\hline \multirow[t]{2}{*}{ Thr } & $\mathrm{ACA} / \mathrm{U} / \mathrm{G} / \mathrm{C}$ & $\mathrm{G}>\mathrm{A}>\mathrm{U}>\mathrm{C}$ & $\mathrm{A}: 28.32(0.17) / \mathrm{C}: 28.16(0.02)$ \\
\hline & & & $\mathrm{G}: 28.34(0.21) / \mathrm{U}: 28.17(0.11)$ \\
\hline \multirow[t]{2}{*}{ Trp } & UGG & $\mathrm{G}>\mathrm{A}>\mathrm{C}>\mathrm{U}$ & $\mathrm{A}: 27.46(0.54) / \mathrm{C}: 27.14(0.24)$ \\
\hline & & & $\mathrm{G}: 27.48(0.62) / \mathrm{U}: 27.01(0.28)$ \\
\hline \multirow[t]{2}{*}{ Tyr } & $\mathrm{UAU} / \mathrm{C}$ & $\mathrm{A}>\mathrm{C}>\mathrm{G}>\mathrm{U}$ & A:28.44(0.36)/C:27.76(0.06) \\
\hline & & & G:27.63(0.30)/U:27.51(0.14) \\
\hline \multirow[t]{2}{*}{ Val } & $\mathrm{GUA} / \mathrm{U} / \mathrm{G} / \mathrm{C}$ & $\mathrm{C}>\mathrm{U}>\mathrm{G}>\mathrm{A}$ & $\mathrm{A}: 27.32(0.25) / \mathrm{C}: 28.13(0.55)$ \\
\hline & & & $\mathrm{G}: 27.69(0.57) / \mathrm{U}: 28.04(0.18)$ \\
\hline
\end{tabular}

${ }^{a}$ The number in bracket are standard deviation.

从反应机理可以看出, 反应整体受到了核苷的调 控. 最有可能影响反应结果的可能是生成 AA- $N-\mathrm{NMP}$ 的这一步, 不同氨基酸的 AA- $N-N M P$ 稳定性和反应性 不同，决定了不同核苷的加入使得反应的进行程度也不 同. 我们推测, 这可能是因为核苷的碱基与氨基酸的侧 链之间有着类似氢键和范德华力的作用，尽管很微弱， 但却足以影响反应. 也正是因为这种作用力的微弱, 使 得用常规的方法未能检测出反应的差别.

这样的结果说明了氨基酸与密码子或反密码子中 间位的核苷确实存在某种特殊的作用，使得不同的核苷 对氨基酸成肽反应的促进程度不同. 正是这种微弱的作
用力, 可能就是密码子与氨基酸之间的联系形成的关键. 这种实验结果为破解密码子之谜提供了一定的支持.

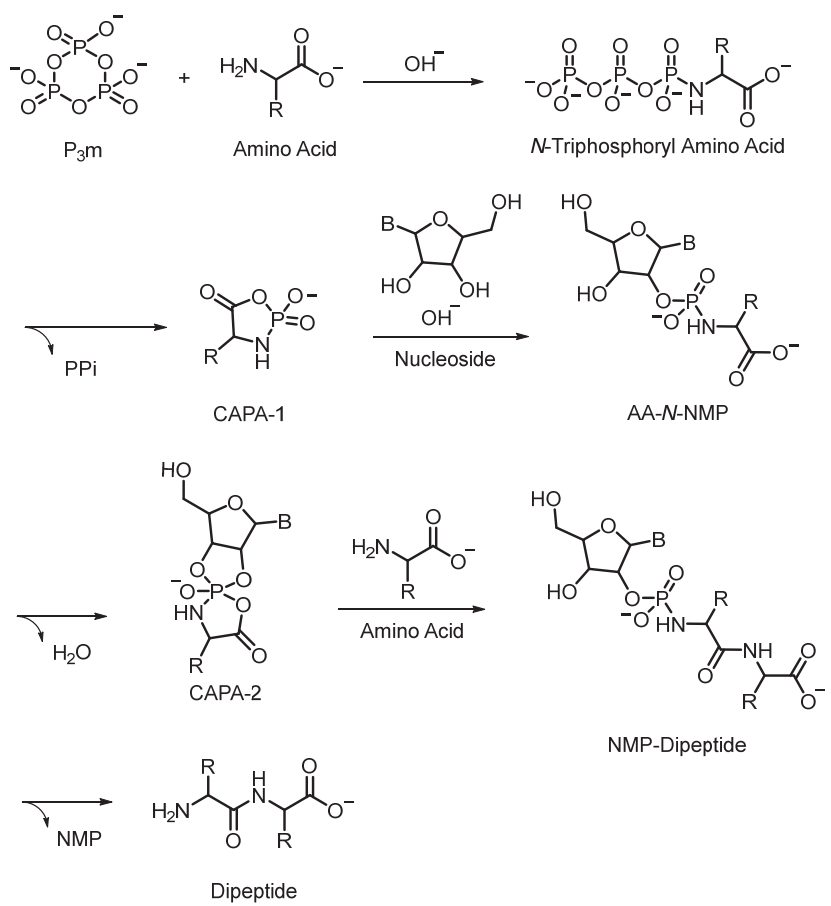

图 1 氨基酸、核苷和三偏磷酸钠反应可能的机理

Figure 1 The plausible mechanism of reaction between amino acid, nucleoside and $\mathrm{P}_{3} \mathrm{~m}$

\section{2 亮氨酸与异亮氨酸的比较}

Leu (CUA, CUC, CUG, CUU) 和 Ile (AUA, AUC, AUU), 这两个氨基酸互为异构体，仅仅是侧链的不同 而已，前者为异丁基，后者为仲丁基，所对应的 AA- $N-N M P$ 也是如此(图 2).<smiles>CCCC(N)C(=O)O</smiles>

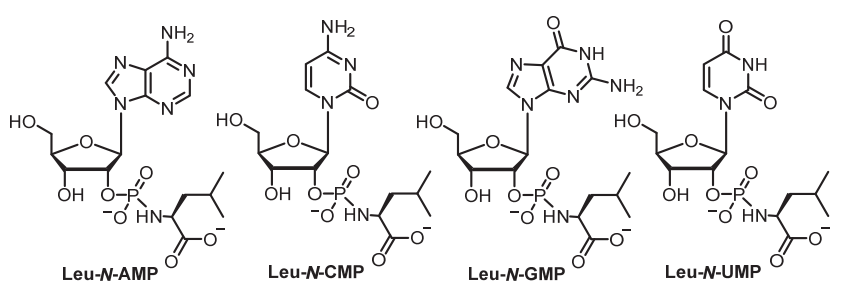

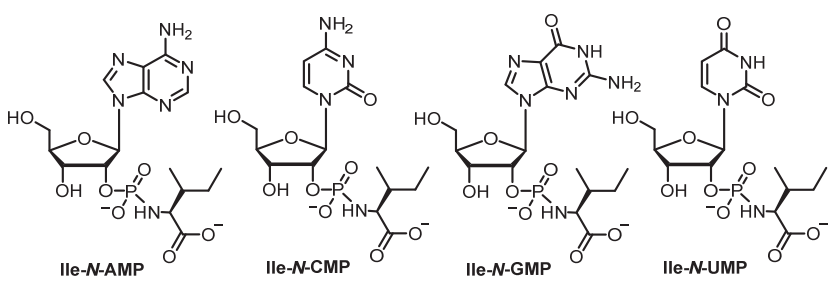

图 2 Leu, Ile 及其对应的 AA- $N$-NMP 的结构

Figure 2 The structures of Leu, Ile and their AA- $N$-NMP 
正常来看, Leu 和 Ile 在反应性上并无明显的差别, 异丁基和仲丁基本身并不带官能团，它们的位阻也相差 不大, 无论是它们各自生成自己对应的 CAPA-1 还是 CAPA-1 继续与核苷反应, 理论上没有明显区别.

然而电子舌测出的结果差别很大, $\mathrm{Leu}$ 为 $\mathrm{G}>\mathrm{U}>\mathrm{A}$ $>\mathrm{C}$, 遵循密码子规则(以变化第二大的碱基为准); Ile 为 $\mathrm{A}>\mathrm{C}>\mathrm{G}>\mathrm{U}$, 遵循反密码子规则, 两者完全没有相似 性. 从这里可以看出氨基酸与密码子或反密码子中间位 的核苷确实存在某种特殊的作用力, 即使氨基酸的 $\mathrm{R}$ 基 发生微小的变化, 也会使得这种作用力发生很大的变 化. 进一步说明密码子识别氨基酸可能与中间位的核苷 与氨基酸的 $\mathrm{R}$ 基产生特殊的作用力有关.

\section{3 结论}

本研究从氨基酸、核苷和三偏磷酸钠的反应出发, 将反应体系前后的变化通过电子舌进行检测, 测出不同 核苷对反应的促进程度的差别. 结果表明, 氨基酸在不 同核苷的调控下, 成肽反应的程度也不同, 往往是其密 码子或反密码子中间位的核苷对其促进作用最大. 从机 理的角度看, 可能是氨基酸的侧链与核苷的碱基之间的 某种弱相互作用造成了这样的结果, 亮氨酸和异亮氨酸 反应结果的差异更加证明了这一点. 正是这种微弱的作 用力, 也许就是目前密码子与氨基酸之间的关系形成的 关键. 本研究结果为破解密码子之谜提供了一定的支 持. 为了进一步证明这种作用力, 后续的研究也在持续 进行中.

\section{4 实验部分}

\section{1 仪器与试剂}

苏氨酸和天冬酰胺购自于上海安耐吉化学公司. 三 偏磷酸钠购自于西格玛公司. 氢氧化钠购自于中国医药 集团有限公司. 其余的氨基酸和核苷都购自于梯希爱. 所有氨基酸都为 $L$ 构型. 所有试剂都为分析纯(AR). 超 纯水 $(18.2 \mathrm{M} \Omega \cdot \mathrm{cm})$ 为实验室自制.

电子舌(浙江工商大学智能感官科学实验室) ${ }^{[16]}$. 超 纯水系统(Milli-Q), Synergy Millipore 公司. 电子天平
(AL104)和实验室 $\mathrm{pH}$ 计(FE20), 梅特勒-托利多仪器(上 海)有限公司. 超声波清洗器(KQ-5200E 和 KQ116), 昆 山市超声仪器有限公司. 恒温水浴锅(DIC-8D, 上海精 宏实验设备有限公司).

\section{2 实验及检测步骤}

将氨基酸、核苷、三偏磷酸钠和氢氧化钠用超纯水 配置成 $\mathrm{pH}$ 为 11 的 $15 \mathrm{~mL}$ 的溶液, 除氢氧化钠外每个组 分的浓度为 $0.0008 \mathrm{~mol} / \mathrm{L}$. 同一反应平行重复三次. 反 应在氮气保护下进行, 于 $50{ }^{\circ} \mathrm{C}$ 反应 $48 \mathrm{~h}$. 反应完后冷 却到室温, 然后用电子舌检测分析, 控制室温在 $25{ }^{\circ} \mathrm{C}$ 左右. 同时每组样品设置一个空白(不加核苷)的原点组 用于对照, 配置完成后立即测试, 测试结果即为样品未 反应的情况. 将原点的数据和反应后的数据一起处理可 计算出样品反应前后的差别. 计算原理和方法采用欧氏 距离平均值(Dis3 值).

\section{References}

[1] Nirenberg, M. W.; Matthaei, J. H. Proc. Natl. Acad. Sci. 1961, 47, 1588.

[2] Crick, F. H. C. J. Mol. Biol. 1968, 38, 367.

[3] Woese, C. R.; Dugre, D. H.; Saxinger, W. C.; Dugre, S. A. Proc. Natl. Acad. Sci. 1966, 55, 966.

[4] Wong, J. A. Proc. Natl. Acad. Sci. 1975, 72, 1909.

[5] Di Giulio, M. J. Theor. Biol. 1998, 191, 191.

[6] Eigen, M.; Gardiner, W.; Schuster, P. Sci. Am. 1981, 244, 88.

[7] Lagerkvist, U. Proc. Natl. Acad. Sci. 1978, 75, 1759.

[8] Ni, F.; Gao, X.; Zhao, Z. X.; Huang, C.; Zhao, Y. F. Eur. J. Org. Chem. 2009, 3026.

[9] Ying, J. X.; Fu, S. S.; Li, X.; Feng, L. B.; Xu, P. X.; Liu, Y.; Gao, X.; Zhao, Y. F. Chem. Commun. 2018, 54, 8598.

[10] Ying, J. X.; Lin, R. C.; Xu, P. X.; Wu, Y. L.; Gao, X.; Zhao, Y. F. Sci. Rep. 2018, 8, 1.

[11] Ying, J. X.; Ding, R. W.; Liu, Y.; Zhao, Y. F. Chin. J. Chem. 2021, 39, 2264.

[12] Da, H.; Sun, Q. Y.; Su, K. Q.; Wan, H.; Li, H. B.; Xu, N.; Sun, F.; Zhuang, L. J.; Hu, N.; Wang, P. Sens. Actuators, B 2015, 207.

[13] Pérez-Ràfols, C.; Sereano, N.; Ariño, C.; Esteban, M.; Díaz-Cruz, J. M. Sensors 2019, 19, 4261.

[14] Jiang, H. Y.; Zhang, M.; Bhandari, B.; Adhikari, B. Food Rev. Int. 2018, 34, 746.

[15] Dong, W. J.; Hu, R. S.; Long, Y. Z.; Li, H. H.; Zhang, Y. J.; Zhu, K. X.; Chu, Z. Food Chem. 2019, 272, 723.

[16] Tian, S. Y.; Deng, S. P.; Chen, Z. X. Sens. Actuators, B 2007, 123, 1049.

(Cheng, B.) 